\title{
Importancia de la nocturia y su impacto en la calidad del sueño y en la calidad de vida en el paciente con hiperplasia benigna de próstata
}

\author{
Hernández Fernández $\mathrm{C}^{1}$, Ristol Pont $\mathrm{J}^{2}$, Estivill E${ }^{3}$, Batista Miranda $\mathrm{JE}^{4}$, López Aramburu MA ${ }^{5}$ \\ ${ }^{1}$ Servicio de Urología. Hospital Universitario Gregorio Marañón. Madrid. ${ }^{2}$ Servicio de Urología. Hospital Mútua \\ de Terrassa. Terrassa. ${ }^{3}$ Unidad de Alteraciones del Sueño. Institut Universitari Dexeus. Barcelona. \\ ${ }^{4}$ Unidad de Urodinamia. Centro Médico Teknon. Barcelona. ${ }^{5}$ Servicio de Urología. Hospital Santiago Apóstol. \\ Miranda de Ebro. Burgos.
}

Actas Urol Esp. 2007;31(3):262-269

\section{RESUMEN}

IMPORTANCIA DE LA NOCTURIA Y SU IMPACTO EN LA CALIDAD DEL SUEÑO Y EN LA CALIDAD DE VIDA EN EL PACIENTE CON HIPERPLASIA BENIGNA DE PRÓSTATA

Introducción: La nocturia es la principal causa de insomnio o de interrupción del sueño en varones adultos, ello se traduce en un efecto negativo sobre la actividad diaria, la calidad de vida y la calidad del sueño.

La valoración de la nocturia y su impacto en la Calidad de Vida (CdV) y en la Calidad del Sueño (CdS) en el paciente con HBP ha sido escasa desde el punto de vista de la investigación clínica, además de la falta de métodos específicos que evaluasen dicho impacto.

Objetivos: Entre los objetivos del tratamiento de la HBP debería procurarse la mejora de la calidad de vida del paciente mediante el control de los síntomas tanto de día como de noche.

Recientemente se han desarrollado instrumentos específicos para valorar la afectación de la nocturia en la CdV, como es el cuestionario N-QoL, así como la afectación en la CdS, por medio de las horas de sueño ininterrumpido (HUS). Sería interesante evaluar cómo la reducción de la nocturia originada por el tratamiento sobre los STUI/HBP puede influir en parámetros objetivos como las HUS, y cómo se traduce en una mejora de la calidad del sueño y de la calidad de vida. Esta valoración debería realizarse, con la ayuda de estas herramientas específicas, durante el desarrollo clínico de los tratamientos farmacológicos.

Con objeto de abordar la nocturia como problema urológico en los pacientes con STUI/HBP, así como de conocer la fisiología del sueño y el efecto de la nocturia sobre el sueño y sobre la calidad de vida, se celebró en Madrid una reunión de expertos urólogos que congregó a una cincuentena de profesionales de toda España. Este artículo presenta los principales conceptos expuestos en esta reunión.

Conclusiones: La nocturia es un síntoma de alta prevalencia en la población masculina de edad avanzada con STUI / HBP. El estudio PreNoc mostró una prevalencia de nocturia en España del 83\% en pacientes $\geq 60$ años diagnosticados de HBP. La nocturia es el síntoma más molesto en los pacientes con STUI/HBP.

Palabras clave: Sintomas del tracto urinario inferior. Hiperplasia benigna de próstata. Nocturia. Prevalencia. Sueño. Calidad de vida.

\section{IMPORTANCE OF NOCTURIA AND ITS IMPACT ON QUALITY OF SLEEP AND QUALITY OF LIFE IN PATIENT WITH BENIGN PROSTATIC HYPERPLASIA.}

Introduction: Nocturia is the main cause of insomnia or sleep interruption in adult men, which has a negative impact on daily activities, quality of life (QoL) and quality of sleep (QoS).

The assessment of nocturia and its impact on $\mathrm{GoL}$ and $\mathrm{GoS}$ in patients suffering from benign prostatic hyperplasia (BPH) has been poor in terms of clinical research, moreover there is a lack of specific methods to assess this impact.

Objetives: The objectives of BPH treatment should include the improvement of patient's QoL by controlling both diurnal and nocturnal symptoms. In order to assess how nocturia affects $\mathrm{BoL}$ and also $\mathrm{BoS}$, some specific tools, such as N-QoL questionnaire or the number of Hours of Undisturbed Sleep (HUS), have been recently developed. Therefore, it would be interesting to assess how nocturia reduction due to LUTS/BPH treatment can impact on some objective parameters such as HUS and also how nocturia reduction improves $\mathrm{GoS}$ and $\mathrm{GoL}$. This assessment should be developed during the application of pharmacological treatments in clinical practice by means of these specific tools.

With the aim of tackling nocturia as an urologic problem in patients with LUTS/BPH, as well as knowing the physiology of sleep and the effect of nocturia on the sleep and BoL, a meeting of expert urologists, that gathered about fifty specialists of all around Spain, was held in Madrid. This article presents the main ideas and concepts exposed in this meeting.

Conclusions: Nocturia is a symptom with a high prevalence in older patients with STUI/BPH. The PreNoc study has showed a Nocturia prevalence in Spain of $83 \%$ in patients $\geq 60$ years old diagnosed of BPH. Nocturia is the most bothersome symptom in patients with STUI/BPH.

Keywords: Lower urinary tract symptoms. Benign prostatic hyperplasia. Nocturia, prevalence. Sleep. Quality of life. 
$L^{\infty}$ os síntomas del tracto urinario inferior (STUI) y más concretamente los sugestivos de hiperplasia benigna de próstata (HBP) son una condición frecuente en varones de edad avanzada ${ }^{1}$. De hecho la prevalencia de HBP aumenta de forma progresiva con la edad. Un estudio epidemiológico realizado en España mostró una prevalencia global del 11,7\% en varones mayores de 40 años, incrementándose hasta el $30 \%$ en pacientes mayores de 70 años ${ }^{2}$. De acuerdo con el consenso sobre terminología de la ICS (International Continence Society), los STUI se dividen en tres grupos: sintomas de llenado, sintomas de vaciado y sintomas postmiccionales ${ }^{3}$. A pesar de que los síntomas de vaciado (dificultad para orinar, flujo urinario débil e intermitente, goteo terminal) son los más prevalentes, habitualmente, los pacientes con HBP manifiestan que los sintomas de llenado (frecuencia de micción, nocturia, urgencia e incontinencia urinaria) son los más molestos $^{4,5}$. De hecho los síntomas de llenado interfieren en mayor medida en las actividades diarias del paciente $\mathrm{y}$, por tanto, tienen un impacto negativo mayor en la calidad de vida de los pacientes con $\mathrm{HBP}^{4}$.

De entre los síntomas de llenado cabe destacar la nocturia, que ya se está considerando como un desorden urinario con entidad propia, más que como sólo un sintoma relacionado con una patología 4 . En este sentido, se llevó a cabo en el año 2006 el estudio PreNoc, promovido por Astellas Pharma, para conocer la prevalencia de la nocturia en España en pacientes $\geq 60$ años diagnosticados de HBP, así como su impacto en la calidad de vida de estos pacientes. A raíz de la presentación de los resultados del estudio PreNoc y con objeto de abordar la nocturia como problema urológico en los pacientes con STUI/HBP, asî como de conocer la fisiología del sueño y el efecto de la nocturia sobre el sueño y sobre la calidad de vida, el 7 de octubre de 2006 se celebró en Madrid una reunión de expertos urólogos que congregó a una cincuentena de profesionales de toda España. Este artículo presenta los principales conceptos expuestos en esta reunión.

La International Continence Society (ICS) define la nocturia como el trastorno que precisa que el paciente se despierte durante la noche una o más veces por la noche para orinar ${ }^{3}$. A pesar de ello, la mayoría de los trabajos epidemiológicos que se han llevado a cabo sobre prevalencia de nocturia en la población definen el sintoma como la necesidad de levantarse 2 o más veces por la noche a orinar, puesto que el paciente reporta que es el hecho de levantarse 2 o más veces lo que se considera molesto. Las cifras de prevalencia de nocturia se estiman entre el 9\% y el 16\% de la población adulta según los estudios ${ }^{4,6}$. A mayor edad de la población de estudio, mayor es la prevalencia de nocturia, pudiendo llegar hasta el 32,4\% en aquellos pacientes de 60 o más años de edad $7,8,9$. De acuerdo con Yoshimura et al, en relación a la enfermedad que nos ocupa, la prevalencia de nocturia en individuos con HBP asciende al $71,1 \%{ }^{10}$. Según los resultados del estudio PreNoc, la prevalencia de nocturia en España en pacientes $\geq 60$ años diagnosticados de HBP es del 83\% [IC95\%: 79,8-86,4].

La nocturia es la principal causa de interrupción del sueño en los varones adultos, lo cual se traduce en un efecto negativo sobre su actividad diaria y, por ello, sobre su calidad de vida 4,6,8. Cuanto mayor es el número de veces que debe levantarse un individuo a orinar por la noche, más negativamente influye sobre su calidad de vida. El sueño juega un papel básico en el bienestar físico y mental del individuo, por lo que la distorsión del sueño que cause la nocturia puede tener consecuencias sobre la salud ${ }^{6,7}$. A pesar de ello muchos de los pacientes que la padecen, no demandan tratamiento para la misma. Éstos adaptan su estilo de vida y su actividad diaria a la nocturia, asumiendo que es una consecuencia de la edad.

El efecto de la nocturia en el sueño y en la calidad de vida puede ser fundamental ante determinadas opciones de tratamiento o de intervención ${ }^{4}$. Cualquier mejora sobre la nocturia repercutirá en una mejora de la calidad del sueño $y$, por ello, en la calidad de vida del paciente ${ }^{6}$.

\section{TERMINOLOGÍA Y CONCEPTOS DEL TRACTO URINARIO INFERIOR: NOCTURIA}

Una de las principales contribuciones de la International Continence Society (ICS) a través de sus trabajos publicados desde 1976 hasta la última publicación en el año $2002^{3}$, ha sido estable- 
cer una estandarización de la terminología médica adecuada a la sintomatología del tracto urinario inferior (STUI). Esta publicación recoge las definiciones de síntomas, signos, observaciones urodinámicas y condiciones asociados a la disfunción del tracto urinario inferior y estudios urodinámicos para su aplicación en todo tipo de pacientes, adultos y pediátricos ${ }^{3}$. Siguiendo esta línea de trabajo, el Grupo Español de Urodinámica y de SINUG publicaron en el año 2005 el consenso sobre terminología y conceptos de la función del tracto urinario inferior ${ }^{11}$, se trata del informe de estandarización de la terminología y conceptos de la función del TUI en castellano. En este documento se usa el término "nocturia" frente al más clásico de "nicturia". Pueden considerarse equivalentes, pero vale la pena seguir las recomendaciones del consenso.

Este documento permite al profesional el uso de un lenguaje preciso para poder referirse a las distintas situaciones clínicas incluyendo, con ello, aquellas que tradicionalmente se consideraban difíciles de definir. De este modo, se consigue una mejor clasificación del paciente, permitiendo un mejor entendimiento y colaboración entre profesionales, incluso de distintas especialidades, así como la selección de cohortes de pacientes homogéneas de cara a la investigación y el desarrollo de práctica clínica basada en la evidencia ${ }^{12}$.

La nocturia ha sido definida por la ICS como la necesidad de despertarse una o más veces durante la noche para orinar. No obstante, a efectos de investigación, la definición más empleada es la que se refiere a "orinar al menos dos veces por noche" 4 .

Existen otros términos asociados a la nocturia como son: poliuria nocturna y frecuencia nocturna. La poliuria nocturna está presente cuando la proporción nocturna (normalmente durante las ocho horas en las que el paciente permanece en la cama) de orina evacuada en 24 horas aumenta. Es dependiente de la edad. La orina evacuada durante la noche excluye la última micción antes de dormir pero incluye la primera micción de la mañana ${ }^{11}$.

La frecuencia nocturna es la necesidad de orinar una o más veces durante la noche, en el tiempo comprendido desde que el individuo se acuesta con intención de dormir y el momento del des- pertar con la intención de levantarse. La nocturia se diferencia en que considera solamente el tiempo en que el individuo está durmiendo ${ }^{11}$.

Cada vez es más común el considerar a la nocturia como un trastorno urinario con entidad propia más que como un síntoma de una enfermedad subyacente ${ }^{4}$. La etiología de la nocturia es multifactorial aunque en la mayoría de pacientes está estrechamente ligada a los STUI producidos por la obstrucción prostática benigna ${ }^{4,13}$. De hecho la nocturia puede estar asociada a entidades como la apnea del sueño, algunas enfermedades somáticas (p. ej. diabetes, arritmias y secuelas de accidentes cerebrovasculares) o factores yatrogénicos como el uso de diuréticos como tratamiento antihipertensivo ${ }^{4,14}$. La incidencia y la severidad de la nocturia aumentan con la edad: en mayores de 80 años cerca del $79 \%$ de los individuos comunican padecer este sintoma $^{15}$. En las personas de edad avanzada, generalmente, la producción de orina en 24 horas es normal pero el ritmo circadiano de la diuresis se modifica de modo que se origina un aumento en la producción nocturna de orina (el volumen de orina durante la noche puede ser igual que el producido durante el día o incluso mayor). Así pues, considerando la elevada prevalencia de HBP en el anciano, confluyen varios factores que explican la relación existente entre nocturia y edad avanzada ${ }^{4,16}$. Independientemente de la causa que la explique, la nocturia se relaciona con insomnio y sueño fragmentado. De hecho se considera que la nocturia es una de las causas más importantes de alteración del sueño en el varón de edad avanzada, siendo la afectación del sueño más severa cuanto mayor es el número de micciones por noche (Fig. 1) 7,17 .

\section{NOCTURIA: EFECTOS SOBRE EL SUEÑO Y LAS ACTIVIDADES COTIDIANAS}

El sueño ha sido una entidad inexplorada por el hombre hasta hace 50 años. Los estudios realizados hasta el momento nos han mostrado que durante el sueño se producen procesos de reparación y restauración, necesarios para la salud física y mental, sabemos que es necesario para el correcto funcionamiento de los sistemas endocrino, metabólico e inmune, pero sin embargo, la 


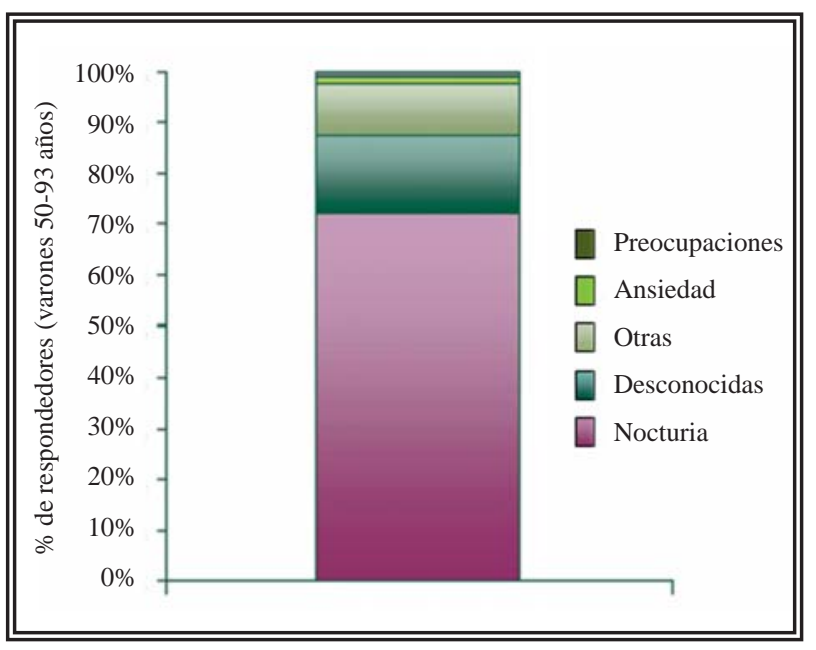

FIGURA 1. Causas de interrupción de la continuidad del sueño en varones de 50-93 años de edad ${ }^{7}$.

función exacta del sueño, todavía resulta desconocida $^{18}$. Para comprender las consecuencias de la nocturia en el sueño es necesario conocer su fisiologia.

El ciclo biológico sueño-vigilia sigue un ritmo circadiano, de aproximadamente 24 horas. El periodo de sueño presenta dos fases diferentes: el sueño REM (del inglés Rapid Eye Movement) y el sueño No REM (NREM). El primero representa el $25 \%$ del tiempo de sueño total y el segundo el $75 \%$ restante ${ }^{19,20}$. La fase REM parece estar relacionada con la función mental y por ello es necesaria para el descanso psicológico, emocional y para la memoria. La fase No-REM estaría ligada a los procesos homeostásicos e implicada con el descanso físico. La fase NoREM se divide en cuatro estados: estado 1, la de transición de la vigilia al sueño, estado 2 , la del sueño ligero y estados 3 y 4 (de sueño profundo) son especialmente importantes para un sueño reparador. La distribución de estas etapas durante el sueño sigue una secuencia común en la mayoría de individuos sanos, las cuales quedan bien reflejadas en el histograma normal del sueño (Fig. 2). Un sueño normal es una sucesión de 3-6 ciclos de sueño. Cada ciclo sueño normal. consta de una sucesión de los cuatro estados de la fase NREM seguidos de la fase REM, con una duración de 90-120 min. Si dividimos la noche en 2 mitades, durante la primera mitad predomina la fase NREM, mientras que durante la segunda predomina la fase $\operatorname{REM}^{18,19,20}$.

Mientras que, en condiciones normales, el despertar natural se produce durante o tras el sueño REM, si la interrupción del sueño se produce durante las fases 3 y 4 del sueño No-REM, ésta causa fatiga, aumento de la sensación de malestar e incluso un descenso del umbral del dolor $^{19}$. Los varones mayores con nocturia se quejan de baja calidad del sueño, aumento de la fatiga diurna, menor grado de bienestar global y comunican un estado de salud peor que los que no padecen nocturia ${ }^{19}$. El porcentaje de individuos que refieren tales molestias aumenta con la severidad de la nocturia ${ }^{19}$. La interrupción del sueño interfiere en la concentración, en el estado de ánimo y en la realización de las actividades cotidianas; la nocturia y la deprivación del sueño pueden conducir también a la depresión o a una mayor susceptibilidad a ciertas enfermedades cardiovasculares $^{6,7,19}$ (Tabla 1). La fatiga y la pérdida de concentración conllevan un aumento del riesgo de accidentes laborales y/o de tráfico, así como caídas y fracturas en ancianos por deambulación a oscuras, cuyo tratamiento aumenta los costes de asistencia sanitaria y cuyas consecuencias pueden ser la pérdida de la independencia física del anciano ${ }^{7,19}$. Así pues la nocturia

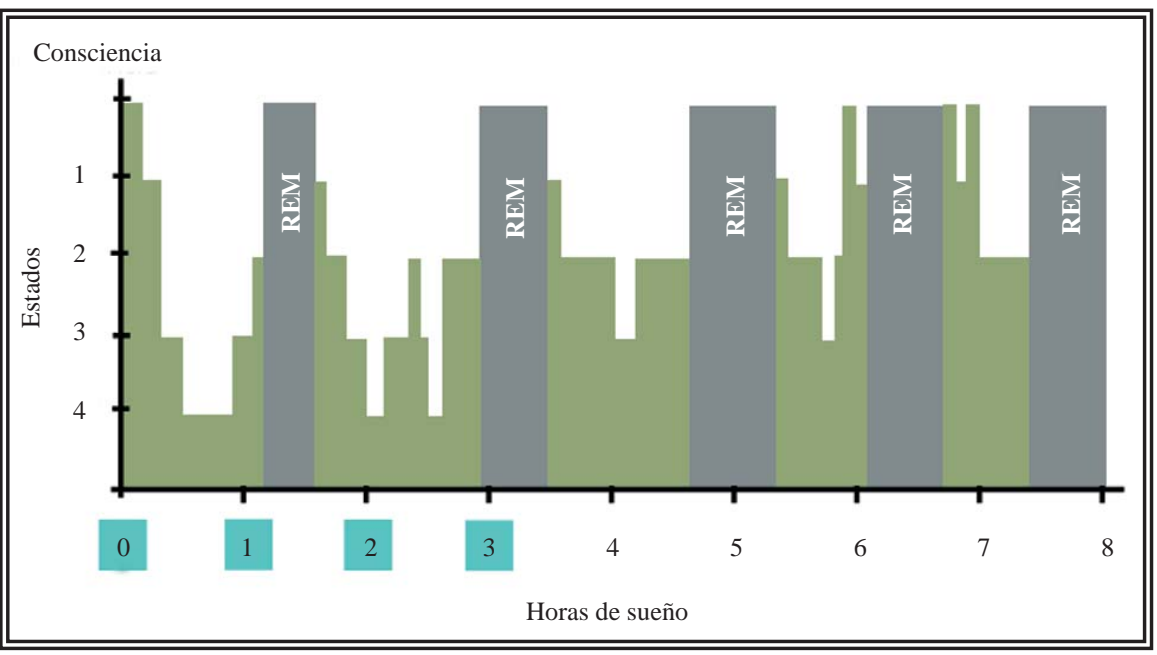

FIGURA 2. Distribución normal de las fases No-REM y REM durante el periodo de 
Tabla 1

Consecuencias de la fragmentación y la deprivación del sueño asociadas a nocturia ${ }^{7}$

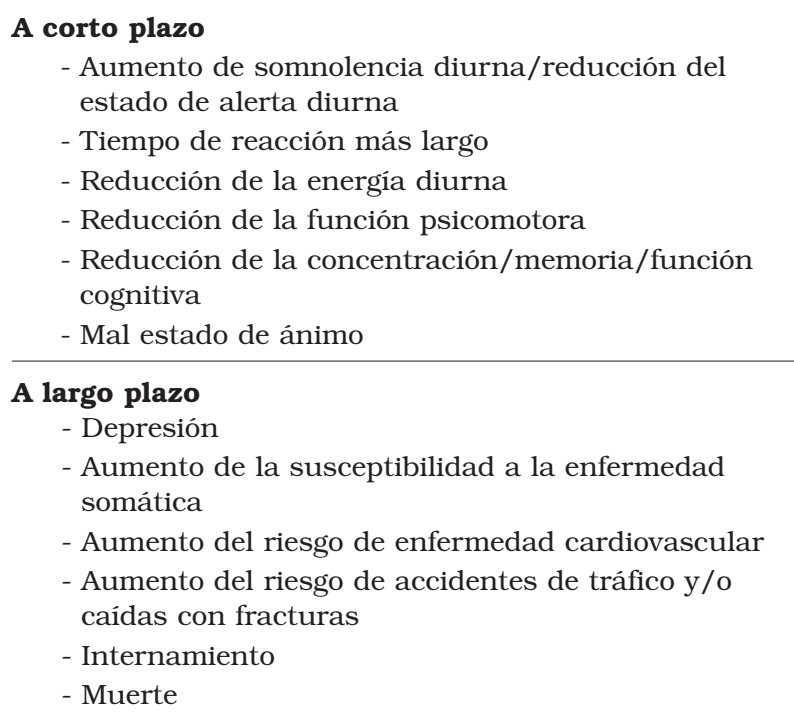

no tiene consecuencias sólo personales y a nivel familiar, sino que también afecta a la sociedad por ser causa de una reducción de la productividad laboral en individuos que por lo demás están sanos $^{7}$.

La edad es un factor de riesgo para el deterioro del sueño ya que induce una disminución de los ritmos circadianos: disminuye el sueño profundo y también el sueño REM, los ciclos de sueño son menores y hay más despertares durante la noche. Sin embargo, los problemas del sueño en las personas mayores se incrementan por la comorbilidad asociada a la edad. Puede aparecer una situación médica que propicie un mayor número de despertares, por lo que es importante eliminar, o al menos reducir, cualquier factor adicional que pueda afectar al sueño en una población con riesgo de sufrir problemas de sueño ${ }^{21}$.

En este sentido se ha comprobado que la nocturia es la causa más común de interrupción o pérdida del sueño en la persona mayor, con el efecto negativo sobre la salud que esto conlle$\mathrm{va}^{17}$.

Por todo lo expuesto anteriormente, en el impacto de la CdV y CdS no sólo es importante la frecuencia de micción nocturna sino también el momento en que el paciente se levanta para miccionar $^{22}$.

\section{IMPACTO DE LA NOCTURIA EN PACIENTES CON STUI/HBP: NUEVAS RECOMENDACIONES}

Durante la "6th International Consultation on Prostate Cancer and Prostate Diseases" (Paris, Junio de 2005), aproximadamente 300 urólogos participaron en un foro de discusión sobre la necesidad de reevaluación del impacto de la nocturia en pacientes con STUI/HBP. Mediante el sistema de votación interactiva, los urólogos dieron su opinión sobre una serie de cuestiones. Más del $50 \%$ consideró el impacto en la calidad de vida como elemento más importante en la evaluación de los STUI/HBP. La gran mayoría (83\%) consideraba que la alteración del sueño debida a la nocturia es el factor que más afecta a la calidad de vida $^{23}$. También resultó mayoritaria la opinión sobre la necesidad de utilizar nuevos instrumentos para monitorizar el impacto de la nocturia en la calidad del sueño y la calidad de vida: el 86\% estaban de acuerdo o totalmente de acuerdo.

Para discutir el estado y la necesidad de las nuevas recomendaciones en la evaluación del impacto de la nocturia en investigación y práctica clínica, un panel de expertos europeos coordinados por Herman Stoevellar, se reunió en Sheiffield, Reino Unido. Como resultado de esta reunión se formularon 7 conclusiones respecto a la medición de la nocturia y su impacto en la calidad de vida ${ }^{23}$.

1. La nocturia es el síntoma más molesto en los pacientes con STUI/HBP, con impacto en la calidad de vida y en la calidad del sueño.

2. La nocturia es multifactorial, y es importante identificar factores concurrentes o condiciones codeterminantes, como la poliuria nocturna, la medicación y los hábitos de ingesta.

3. Despertarse durante las primeras horas de sueño tiene mayor impacto en la calidad del sueño. Es importante valorar las horas de sueño ininterrumpido (HUS).

4. Los métodos que actualmente se utilizan para medir la nocturia carecen de sensibilidad para valorar el impacto de este sintoma en la calidad de sueño y en la calidad de vida.

5. Los métodos potenciales para evaluar calidad de vida y la calidad del sueño incluyen: diarios miccionales, cuestionario N-GoL y valoración de Horas de Sueño Ininterrumpido. 
6. La evaluación de los nuevos tratamientos para STUI / HBP debería incluir valoración de la nocturia, de la calidad de vida y de las horas de sueño ininterrumpido (mediante diarios miccionales o instrumentos como el actígrafo) .

7. A partir de la investigación clínica, se deberían desarrollar instrumentos válidos y sencillos para uso en práctica clínica diaria.

En resumen, las directrices futuras están encaminadas en la búsqueda de procedimientos sencillos y útiles a partir de métodos complejos. A partir de la simplificación se consigue evaluar mejor a los pacientes y mejorar el perfil de los síntomas y puntos diagnósticos.

\section{INCORPORACIÓN DE NUEVOS INSTRUMENTOS ESPECÍFICOS DE VALORACIÓN DE LA NOCTURIA}

Uno de los objetivos principales del tratamiento de la HBP debería ser mejorar la calidad de vida del paciente mediante el control de los síntomas tanto de día como de noche.

Existe claramente un vacío de métodos específicos, sensibles y validados que valoren el impacto de la nocturia sobre la CdV y la CdS.

La valoración de la nocturia y su impacto en la CdS y CdV en el paciente con HBP ha sido escasa desde el punto de vista de la investigación clínica. En el terreno de los STUI/HBP, la variable de eficacia más importante incluida para el estudio de la eficacia de un tratamiento tanto en los ensayos clínicos con tratamiento farmacológico como en los de otro tipo de intervenciones invasivas ha sido tradicionalmente el cuestionario International Prostate Symptom Score (I-PSS) ${ }^{7,24}$. Los únicos datos disponibles actualmente acerca de la nocturia son los relativos a la pregunta que sobre este síntoma se incluye en el cuestionario I-PSS. A pesar de que esa pregunta da poca información, se ha comprobado recientemente en nuestro entorno que la nocturia sigue siendo el sintoma que más impacta en la calidad de vida, incluso si medimos ésta con un cuestionario general como es el FACT ${ }^{25}$. Salvo el recientemente desarrollado cuestionario N-QoL, no existen instrumentos que valoren de manera específica el impacto de la nocturia sobre la calidad de vida del paciente ${ }^{4}$.
Las horas de sueño ininterrumpido o HUS (del inglés, Hours of Undisturbed Sleep) definidas como el tiempo transcurrido desde que el paciente se queda dormido hasta que se levanta por primera vez a orinar, es un parámetro potencialmente importante desarrollado para evaluar la afectación de la calidad del sueño y de la calidad de vida debido a la nocturia y, por tanto, para evaluar un tratamiento frente a los STUI/HBP.

En este sentido existe un estudio piloto en fase IIIb para determinar el efecto de la formulación OCAS de tamsulosina sobre la nocturia, HUS y calidad de vida en pacientes con STUI/HBP. En este estudio se observó una mejoría de la nocturia, un aumento de las horas de sueño ininterrumpido y una mejoría de la Calidad de Vida en los pacientes tratados con Tamsulosina OCAS ${ }^{26}$.

El estudio RESTORE, actualmente en fase de realización, es un estudio pionero en urología puesto que utiliza técnicas establecidas en cronobiología y en estudios del sueño. Una de las herramientas utilizadas para la medida de la nocturia es el actígrafo (actiwatch). El actígrafo es un dispositivo electrónico ligero, para llevar en la muñeca, que mide el sueño y la actividad durante la noche, de manera que permite una recogida de datos más rigurosa y precisa que el registro en diarios en papel y transformar esta información en gráficos de análisis del sueño (Fig. 3). En este estudio se evalúa el efecto de la formulación OCAS de tamsulosina en la mejoría de la frecuencia miccional nocturna, comparado con placebo. Los objetivos secundarios incluyen la evaluación del efecto de la formulación OCAS de tamsulosina en las HUS y en parámetros de CdV asociados a nocturia, parámetros de sueño/energía y molestias/preocupación, así como su eficacia y tolerabilidad.

La falta de estudios centrados en nocturia hacía patente la urgente necesidad de investigaciones adicionales en este terreno. Es interesante evaluar cómo la reducción de la nocturia originada por el tratamiento sobre los STUI/HBP puede influir en las HUS, y cómo se traduce este resultado en una mejora de la calidad de vida. Para ello es necesario el desarrollo de instrumentos específicos. El cuestionario N-QoL, como ya hemos visto, es uno de ellos ${ }^{9}$. Los datos de inves- 


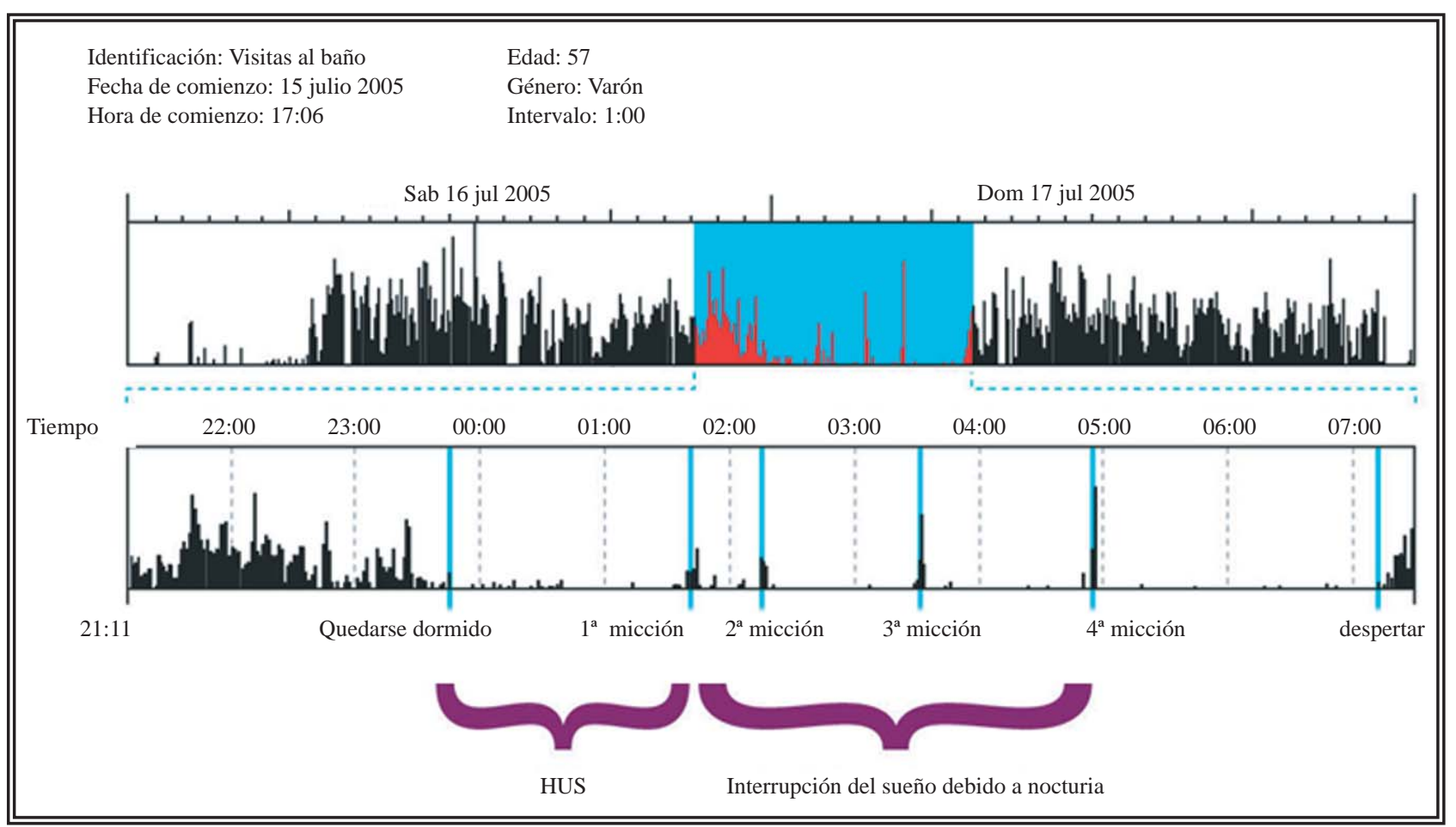

FIGURA 3. Gráfico de análisis del sueño con determinación de horas de sueño ininterrumpido (HUS) obtenido mediante dispositivo electrónico.

tigación obtenidos a través de cuestionarios de sueño/calidad de vida específicos de nocturia y mediante instrumentos avanzados de valoración del sueño proporcionarán una mejor perspectiva de la relación entre nocturia y sueño, los efectos sobre la energía diurna y la calidad de vida, y cómo esto es afectado por el tratamiento ${ }^{6}$.

\section{CONCLUSIONES}

La nocturia es un síntoma de alta prevalencia en la población masculina de edad avanzada con STUI / HBP. El estudio PreNoc mostró una prevalencia de nocturia en España del $83 \%$ en pacientes $\geq 60$ años diagnosticados de HBP. La nocturia es el síntoma más molesto en los pacientes con STUI/HBP. Su impacto en la calidad del sueño se traduce en fatiga diurna, disminución de la función cognitiva y aumento de la susceptibilidad a determinadas enfermedades como la depresión. Todo ello puede llevar a una disminución importante de la calidad de vida del paciente. Es imprescindible que el tratamiento de los STUI/HBP vaya dirigido a aliviar síntomas tales como la nocturia. Los métodos actuales para medir la nocturia no son específicos para valorar el impacto de este sintoma en la calidad del sueño y en la calidad de vida. Se han desarrollado nuevos métodos que pueden ser potencialmente útiles para este tipo de evaluación: el cuestionario $\mathrm{N}-\mathrm{Q}$ oL o la valoración de las horas de sueño ininterrumpido (HUS) para lo que se están evaluando dispositivos electrónicos. Todo ello está dirigido a optimizar el tratamiento de la nocturia y evaluar si, además, se asocia con un aumento de las horas de sueño ininterrumpido. En conclusión, la nocturia y su efecto sobre la calidad del sueño y la calidad de vida reclaman un papel relevante en la valoración del tratamiento en pacientes con HBP. Esta valoración debería realizarse, con la ayuda de herramientas específicas, durante el desarrollo clínico de los tratamientos farmacológicos.

\section{REFERENCIAS}

1. Thorpe A, Neal D. Benign prostatic hyperplasia. Lancet. 2003;361(9366):1359-1367.

2. Chicharro-Molero JA, Burgos-Rodriguez R, Sanchez-Cruz JJ, Rosal-Samaniego JM, Rodero-Carcia P, Rodríguez Vallejo JM. Prevalence of benign prostatic hyperplasia in Spanish men 40 years old or older. J Urol. 1998;159(3):878882. 
3. Abrams P, Cardozo L, Fall M, Griffiths D, Rosier P, Ulmsten $\mathrm{U}$, et al. The standardisation of terminology of lower urinary tract function: report from the Standardisation Subcommittee of the International Continence Society. Neurourol Urodyn. 2002;21(2):167-78.

4. Schulman CC, Asplund R, Desgrandchamps F, Jonas U. The impact of nocturia on health status and quality of life in patients with lower urinary tract symptoms suggestive of benign prostatic hyperplasia (LUTS/BPH). Eur Urol Suppl. 2005;4:1-8

5. Vela-Navarrete R, Alfaro V, Badiella LL, Fernandez-Hernando N. Age-stratified analysis of I-PSS and $\mathrm{BoL}$ values in spanish patients with symptoms potentially related to BPH. Eur Urol. 2000;38(2):199-207.

6. Asplund R. Nocturia: consequences for sleep and daytime activities and associated risks. Eur Urol Suppl 2005;3(6): 24-32.

7. Abrams P. Nocturia: the effect on sleep and related health consequences. Eur Urol Supp. 2005; 3 (6): 1-7.

8. Abraham L, Hareendran A, Mills IW, Martin ML, Abrams P, Drake MJ, et al. Development and validation of a qualityof-life measure for men with nocturia. J Urol. 2004;63(3): 481-486.

9. Asplund R. Nocturia in relation to sleep, health, and medical treatment in the elderly. BJU International. 2005;96 (Suppl 1):15-21.

10. Yoshimura K, Ohara H, Ichioka K, Terada N, Matsui Y, Terai A, et al. Nocturia and benign prostatic hyperplasia. Urology. 2003;61(4):786-790.

11. Grupo Español de Urodinámica y de la SINUG. Consenso sobre terminología y conceptos de la función del tracto urinario inferior. Actas Urol Esp. 2005;29(1):16-30.

12. Artibani W, Novara G. The International Continence Society terminology for the lower urinary tract: the importance of standardization. Nat Clin Pract Urol. 2005;2(12): 576-577.

13. Abrams P. Nocturia: the major problem in patients with lower urinary tract symptoms suggestive of benign prostatic obstruction (LUTS/BPO). Eur Urol Suppl 2005;3(6):816.

14. Asplund R. Nocturia in relation to sleep, somatic diseases and medical treatment in the elderly. BJU Int. 2002;90(6): 533-536.
15. Wein A, Lose GR, Fonda D. Nocturia in men, women and the elderly: a practical approach. BJU Int. 2002;90 (Suppl 3):28-31.

16. Asplund R. Nocturia, nocturnal polyuria and sleep quality in the elderly. J Psychosom Res. 2004;56(5):517-25.

17. Middelkoop HA, Smilde-van den Doel DA, Neven AK, Kamphuisen HA, Springer CP. Subjective sleep characteristics of 1,485 males and females aged 50-93: effects of sex and age, and factors related to self-evaluated quality of sleep. J Gerontol A Biol Sci Med Sci. 1996;51(3):M108-115.

18. Roehrs T. Sleep physiology and pathophysiology. Clin Cornerstone. 2000;2(5):1-15.

19. Stanley N. The Underestimated Impact of Nocturia on Quality of Life. Eur Urol Suppl. 2005;4(7):17-19

20. Keenan SA. Normal human sleep. Resp Clin N Am. 1999 5(3):319-331.

21. Akerstedt T, Billiard M, Bonnet M, Ficca G, Garma L, Mariotti M, et al. Awakening from sleep. Sleep Med Rev. 2002;6(4):267-286.

22. Chartier-Kastler E, Tubaro A. The Measurement of Nocturia and its Impact on Quality of Sleep and Quality of Life in LUTS/HBP. Eur Urol Suppl 2006;5:3-11.

23. Chapple CR, Batista JE, Berges R et al. The Impact of Nocturia in Patients with LUTS/HBP: Need for New Recommendations. Eur Urol Suppl 2006;5:12-18.

24. Batista JE, Regalado R, Chechile G et al, Cuestionario IPSS en pacientes y controles. Validación psicométrica. Act Urol Esp. 1995;19:93-101.

25. Batista JE, Molinuevo B, Pardo Y. (Impact of lower urinary tract symptoms on quality of life using the fact-G scale) Urology 2007, en prensa.

26. Djavan B, Milani S, Davies J, Bolodeoku J. The Impact of Tamsulosin Oral Controlled Absorption System (OCAS) on Nocturia and the Quality of Sleep: Preliminary Results of a Pilot Study. Eur Urol Suppl 2005;4(2):61-68.

Correspondencia autor: Dr. C. Hernández Fernández

Servicio de Urología. Hospital Universitario Gregorio Marañón Dr. Esquerdo 46. 28007 Madrid. Tel.: 915868158

E-mail autor: carloshfdez@telefonica.net

Información artículo: Original - HBP

Trabajo recibido: diciembre 2006

Trabajo aceptado: enero 2007 